Int. J. Dev. Biol. 52: 561-570 (2008)

doi: $10.1387 / \mathrm{ijdb} .072523 \mathrm{ds}$

\title{
Regionalized calcium signaling in zebrafish fertilization
}

\author{
DIPIKA SHARMA and WILLIAM H. KINSEY* \\ Department of Anatomy and Cell Biology University of Kansas Medical Center, Kansas City, Kansas, USA
}

\begin{abstract}
Fertilization involves an initial, highly localized signal delivered by the sperm, which becomes amplified by a signal transduction cascade to impact the entire oocyte cytoplasm. The zebrafish oocyte presents a unique opportunity to study this process since fertilization always occurs at the micropyle, allowing the investigator to image the earliest steps in the oocyte activation process. The objective of the present study was to characterize the amplification of the sperm-induced calcium transient in the zebrafish oocyte and test the role of Fyn kinase in this process. Confocal fluorescence microscopy revealed that the sperm-induced calcium transient was composed of two elements, one of which was unique to the oocyte cortex and a second, slower transient that occurred in the central cytoplasm of the oocyte. The cortical transient was initiated immediately deep to the micropyle, became amplified at the animal pole, and progressed peripherally through the oocyte cortex. This was followed by a slower transient that occurred in the central cytoplasm of the oocyte. Several lines of evidence indicate that calcium release in these two compartments may be regulated differently. The calcium transient in the oocyte cortex is highly sensitive to inhibition by Fyn-SH2 domain containing fusion proteins, while the central cytoplasmic transient is relatively resistant to this treatment. Oocytes stimulated by injection of a soluble extract prepared from zebrafish sperm respond only with a cortical calcium transient initiated at the micropyle, while oocytes stimulated parthenogenetically by hypotonic shock exhibit a defective cortical transient but a normal transient in the central cytoplasm. Analysis of the subcellular distribution of Fyn kinase and the IP3 receptor reveal that these important signaling components are highly enriched in the oocyte cortex, a factor which may facilitate a faster propagation of the calcium transient in this compartment. In summary, analysis of calcium signaling in the zebrafish oocyte requires attention to morphologically distinct compartments of the oocyte and it is likely that these compartments are controlled by different biochemical events.
\end{abstract}

KEY WORDS: fertilization, oocyte, calcium, Fyn, protein kinase, IP3

\section{Introduction}

Fertilization triggers a series of preprogrammed biochemical steps in the oocyte, which function to establish a block to polyspermy, activate zygote metabolism, and initiate development. An early step in this process is the transient elevation of intracellular free calcium. This fertilization-induced calcium transient is common to oocytes of all species examined including organisms as primitive as the sponge and as complex as mammals (Carroll,.2001; Runft, et al., 2002; Swann, et al., 2004). However, significant species differences exist in the subcellular distribution, timing, and biochemical triggers for these calcium transients. Model fertilization systems that were initially used to study fertilization-induced calcium signaling include a wide variety of externally fertilizing species such as starfish, sea urchins, fish, and amphibians. Oocytes of these species tend to respond to fertilization with a single, rapid, high amplitude calcium transient that progresses from the point of sperm-oocyte fusion to include the entire oocyte. In species such as the starfish and sea urchin, the calcium transient is manifested as a fairly uniform wave of free calcium that progresses across all subcellular compartments of the zygote (Carroll, et al.,1997; Galione, et al.,1993). However oocytes from fish and amphibian species are

\footnotetext{
Abbreviations used in this paper: BSA, bovine serum albumin; EDTA, ethylenediaminetetraacetic acid; HEPES, [2-hydroxyethyl] piperazine-N'[2-ethane-sulfonic acid];IP3, Ins 1,4,5 P3; PTK, protein tyrosine kinase; SFK,Src-family protein kinase; $\mathrm{SH} 2$, Src homology domain 2; mpi, minutes post-insemination.
}

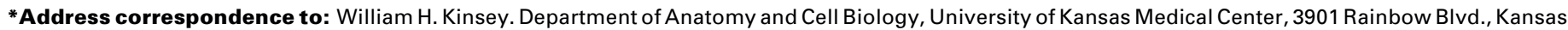
City, KS 66160, USA. Fax: +1-913-588-2710. e-mail: wkinsey@kumc.edu
} 

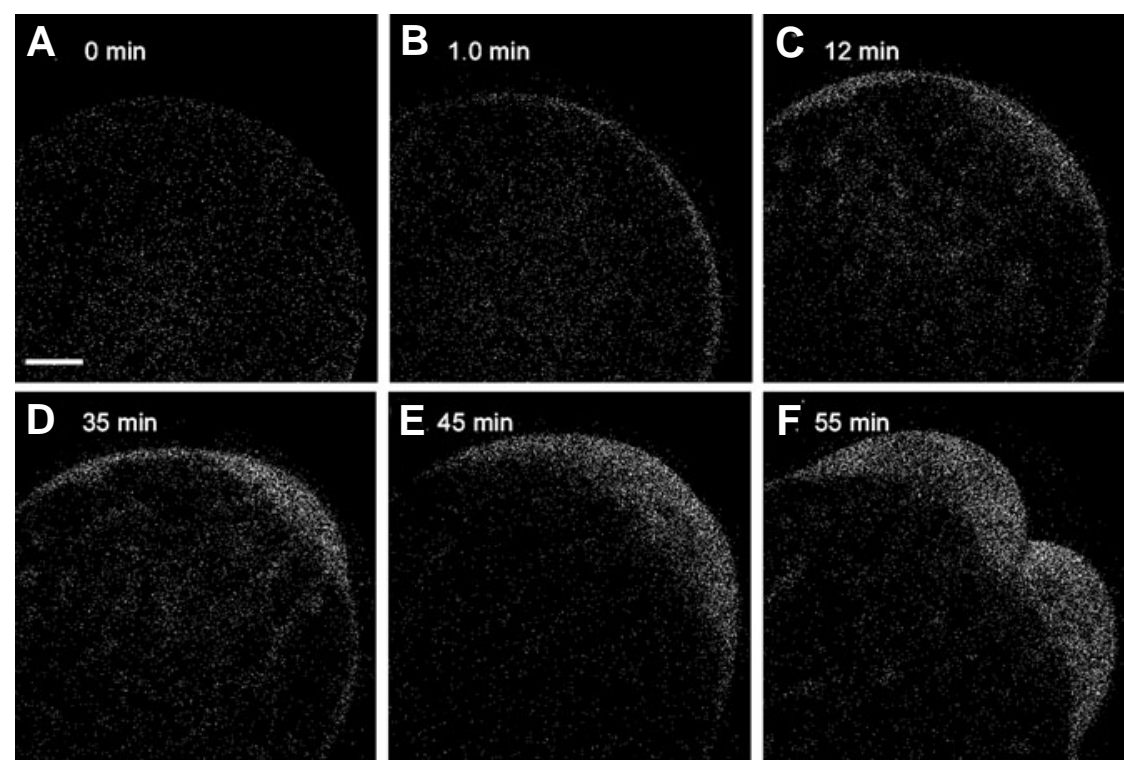

Fig. 1. Calcium release following fertilization of the zebrafish oocyte. Changes in cytosolic free calcium were detected by calcium green-dextran fluorescence imaged by confocal fluorescence. The oocyte was immobilized in a $750 \mu \mathrm{m}$ well with the animal pole oriented to the upper right. Unfertilized oocytes were injected with calcium green dextran to achieve a final intracellular concentration of $500 \mathrm{nM}$, and allowed to recover for 10 min before photography began. Calcium release was initially observed within a thin layer of cortical cytoplasm near the animal pole, and subsequently spread laterally to involve the entire oocyte cortex. The cytoplasm of the blastodisc was highly active in calcium signaling during the later stages of oocyte activation and cleavage. Images were taken with a $4 X$ objective just before addition of sperm + aquarium water, time $=0$ (A) and at different times; $1 \mathrm{~min}$ (B), $12 \mathrm{~min}$ (C), $35 \mathrm{~min}$ (D), $45 \mathrm{~min}$ (E) and $55 \mathrm{~min}(\mathbf{F})$ after insemination. more polarized both with respect to morphology and developmental potential. The highly polarized fish oocyte represents an extreme case in which fertilization can only occur through an opening in the chorion (the micropyle) located near the animal pole of the oocyte and this morphological specialization is also reflected in the properties of the fertilization-induced calcium transient. Fish species examined include medaka (Gilkey, et al.,1978);(Yoshimoto, and Hiramoto,1988);(Iwamatsu, et al.,1988a; Iwamatsu, et al.,1988b), goldfish (Yamamoto,1954), and zebrafish (Iwamatsu, et al.,1988b; Lee, et al.,1999; Webb, et al.,1997) (Lee, et al.,2003) and the results have consistently

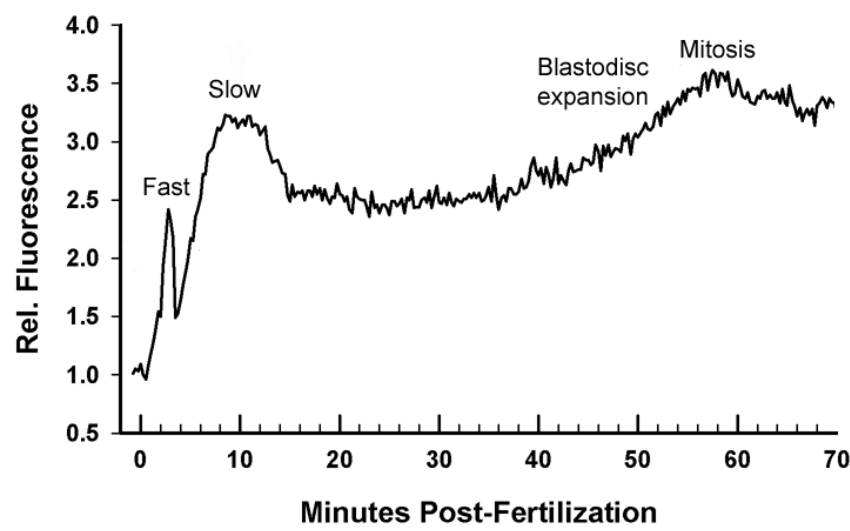

Fig. 2. Quantification of fertilization-induced changes in calcium green fluorescence. An oocyte was injected with calcium green dextran and the control protein GST (5 $\mu \mathrm{M})$, then after a 10 min recovery period, images were recorded every $15 \mathrm{sec}$. Fertilization was initiated by addition of a mixture of sperm and water at time $=0$. Fluorescence intensity across an optical cross section through the equator of the oocyte was measured by pixel intensity quantitation using the area measurement tool from Metamorph 6.1 within a measurement region traced around the periphery of the entire oocyte. Calcium green fluorescence increased rapidly after insemination with two peaks of fluorescence intensity during the first $10 \mathrm{mpi}$. A slower prolonged increase began during blastodisc expansion and peaked at mitosis. shown that fish oocytes also respond to fertilization with an IP3mediated calcium transient which propagates across the entire oocyte. These calcium transients were also shown to involve Srcfamily PTK activation (Kinsey, et al.,2003).

The objective of this report is to examine the fertilization induced calcium events in the zebrafish oocyte and describe biochemical specializations that may account for the localized aspects of these calcium-signaling events. Earlier work documented fertilization induced propagation of calcium release through the zebrafish oocyte and revealed that calcium release in the oocyte cortex occurred more rapidly than in the central cytoplasm (Lee, et al.,1999). We now have made use of confocal imaging techniques to resolve highly localized features of the fertilizationinduced calcium transient and test the requirement for Fyn kinase activity in calcium signaling within these compartments of the oocyte. The results demonstrate that calcium signaling in the cortex of the zebrafish oocyte is highly polarized with respect to the animal pole, and differs from calcium release in the yolky central cytoplasm in that it requires Fyn kinase signaling for optimal propagation. Calcium release in the cortex also requires a fertilizing sperm for optimal propagation while calcium release in the central cytoplasm can be induced by hypotonic shock caused by spawning in the absence of sperm. Therefore, the cortex of the zebrafish oocyte seems to be specialized for a rapid response to the fertilizing sperm, making use of protein tyrosine kinase (PTK) stimulated IP3 production to ensure rapid establishment of a block to polyspermy. The central cytoplasm of the zebrafish oocyte lacks this rapid response.

\section{Results}

Timing and morphology of calcium signaling at fertilization

Fertilization-induced calcium signaling in zebrafish oocytes has been studied by fluorescence microscopy using a calcium sensitive fluorescent protein such as aequorin (Creton, et al.,1998; Lee, et al.,1999). These studies demonstrated that fertilization triggered an initial calcium transient in which the resting calcium level of $60 \mathrm{nM}$ increased to $90-120 \mathrm{nM}$. This was followed by later 
Fig. 3. Calcium signaling during early stages of oocyte activation. The progression of calcium release during the first eight minutes post-insemination was monitored by calcium green fluorescence as described in Fig. 1. The initial calcium release occurred in a small region of cortical cytoplasm, butspread peripherally to include the entire cortex by $8 \mathrm{mpi}$. Calcium release in the central cytoplasm also spread from the animal pole (left) to include the entire oocyte. The time post-insemination is indicated at the top of each panel and magnification is indicated by the bar which represents $100 \mu \mathrm{m}$.
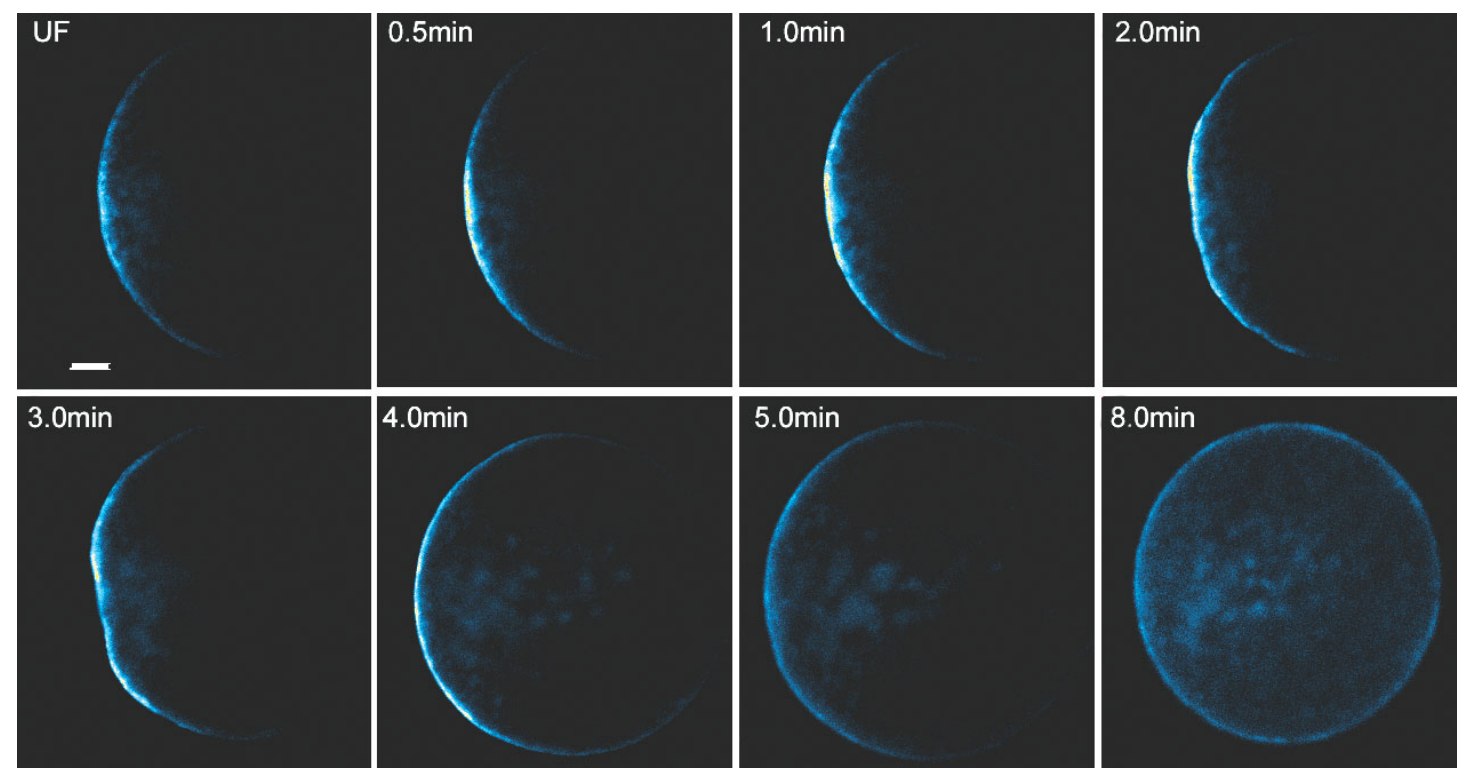

oscillations that were associated with mitosis. Our studies involving the biochemical control of calcium signaling during oocyte activation have used confocal fluorescence microscopy to quantitate calcium changes using calcium green-dextran as a calcium reporter. Confocal imaging has allowed us to quantitate calcium changes with greater spatial resolution than in the earlier studies and the fact that fertilization occurs through the micropyle allowed us to map calcium changes relative to the site of sperm-oocyte contact. For these experiments, unfertilized oocytes were maintained in a quiescent state with an isotonic medium thought to mimic coelomic fluid. The oocytes were then injected with calcium green dextran and fertilization was accomplished by addition of sperm followed by aquarium water, which activated sperm motility. Figure 1 demonstrates the fertilization-induced calcium transient as imaged through an optical cross section of the oocyte. The unfertilized zebrafish oocyte exhibited little evidence of calcium signaling (Fig. 1A), however fertilization triggered a rapid increase in $\left[\mathrm{Ca}^{2+}\right]_{\mathrm{i}}$ as evidenced by enhanced calcium green fluorescence in the oocyte cortex (Fig1B). The fluorescence in the oocyte cortex continued to increase during the first 12-15 minutes post insemination (mpi), particularly at the animal pole (Fig1C). By $35 \mathrm{mpi}$, streaming of cytoplasm to the animal pole was obvious as 'streamers' of calcium green fluorescence leading to the animal pole cytoplasm, which was highly active in calcium signaling (Fig1D). Cytoplasmic streaming resulted in accumulation of cytoplasm at the animal pole, which formed a bulge known as the blastodisc (Fig 1E). Cytokinesis occurred at $55 \mathrm{~min}$ as seen by the presence of a cleavage furrow (Fig1F) and was associated with intense calcium signaling as described (Webb, et al., 1997).

In order to quantitate the changes in calcium green fluorescence, the fluorescence intensity of an optical section through the entire oocyte was measured by Metamorph software and is presented graphically in Fig. 2. Measurements made across the entire oocyte cross section demonstrate that fertilization was followed by an initial low amplitude calcium transient beginning as early as $30 \mathrm{sec}$ after sperm addition and reaching a maximum at 2-4 mpi. The initial transient was followed by a more prolonged transient reaching a higher average amplitude with a maximum at 8-10 mpi. These two initial transients were followed by later calcium signaling events (30-60 mpi) associated with blastodisc expansion and cytokinesis as reported elsewhere (Chang, and Meng, 1995; Lee, et al., 2003).

Closer examination of the early fertilization-induced calcium events is shown in Fig. 3. Here an oocyte held in a suction pipette with the micropyle oriented to the left was fertilized and calcium

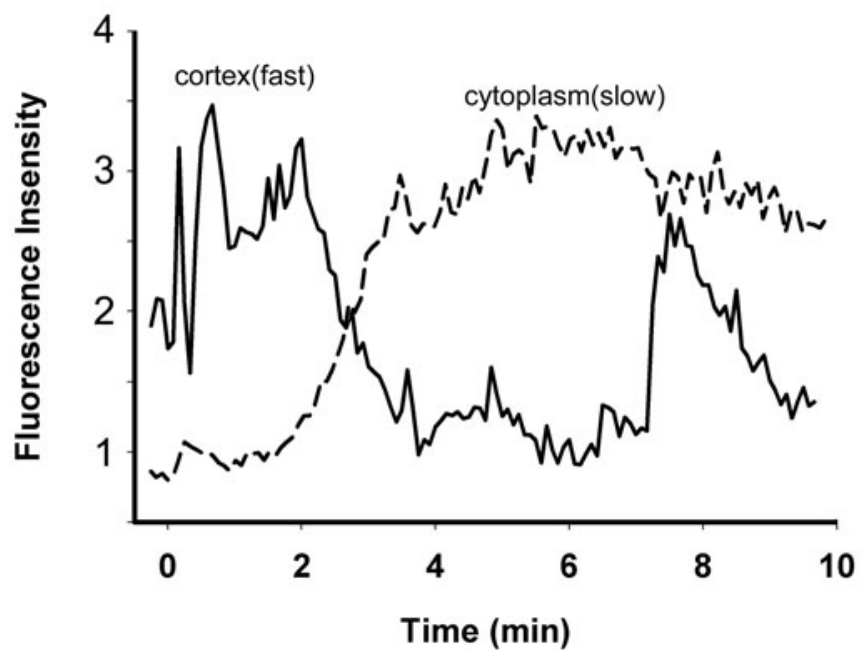

Fig. 4. Quantification of calcium dynamics in subcellular compartments. An oocyte was injected with calcium green-dextran and fertilized as in Fig. 2. Fluorescence intensity was quantitated in regions drawn to include representative areas of the oocyte cortex and central cytoplasm. The dashed line represents fluorescence measured within a circular region in the center of the zygote (central cytoplasm) comprising approximately $25 \%$ of the total cross sectional area. The solid line represents fluorescence measured within an arc traced over the cortex approximately $30 \mu \mathrm{m}$ thick (cortical cytoplasm) as near as possible to the micropyle. This arc comprised approximately $20 \%$ of the perimeter of the zygote. The solid line demonstrates that calcium release in the cortes occurs more rapidly than that in the central cytoplasm. 

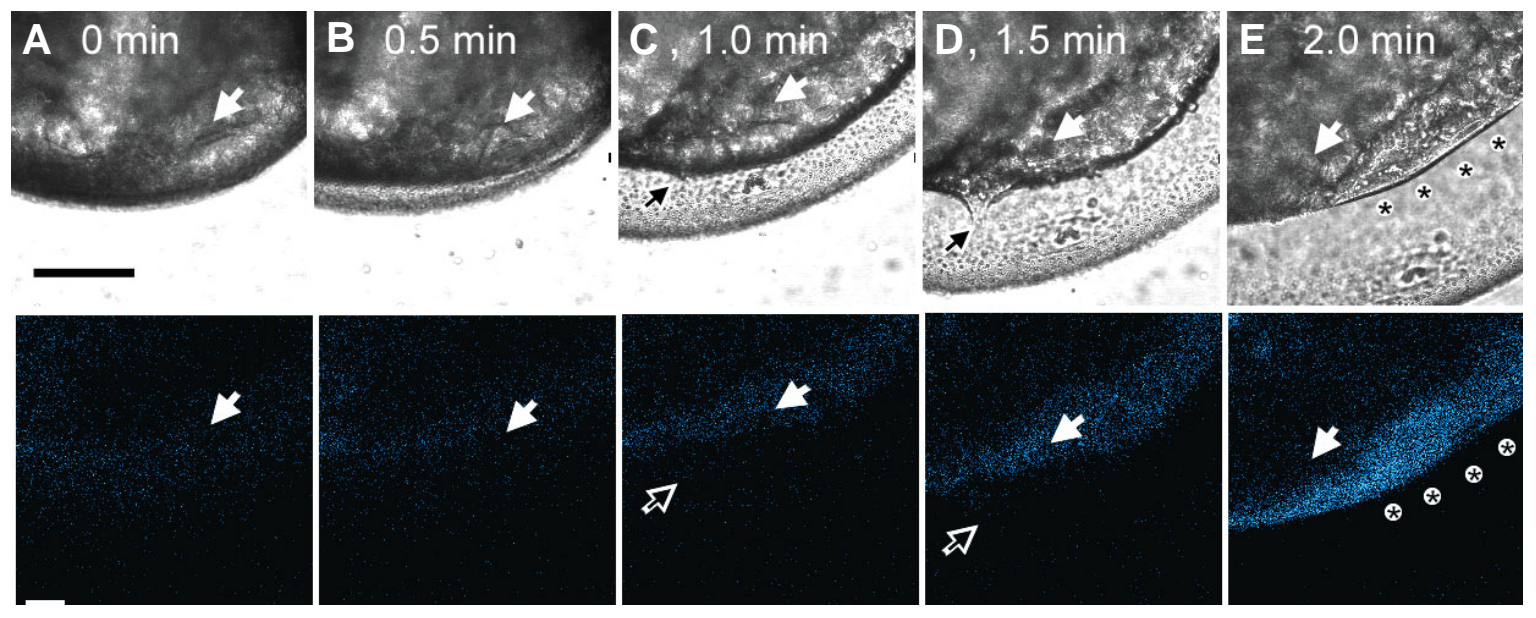

Fig. 5. Calcium signaling at the site of sperm incorporation. The progression of calcium release at the micropyle was followed by using a suction pipette to position an oocyte such that the micropyle was visible. Calcium green dextran was then injected from the left and after a 10-min recovery period, images from transmitted light (upper panels) and calcium green fluorescence (lower panels) were recorded at 15 sec intervals. Unfertilized oocyte (A), $0.5 \mathrm{mpi}$ (B), $1.0 \mathrm{mpi}$ (C), $1.5 \mathrm{mpi}$ (D) and $2.0 \mathrm{mpi}$ (E). The position of the depression in the oocyte cortex caused by the micropyle is indicated by the white arrow. The fertilization cone is indicated by the black arrow. The clear cytoplasm at the animal pole which became apparent at 2.0 mpi is indicated by $\left(^{*}\right)$. The initial calcium release at the site of sperm fusion is barely recognizable in (B), but obvious in (C). It is also apparent that the initial calcium response at the site of sperm-egg interaction is strongly amplified once it reached the animal pole. Magnification is indicated by the bar, which represents $25 \mu \mathrm{m}$.

fluorescence was recorded for the first eight min. Prior to fertilization, the cortex overlying the animal pole exhibited some elevated calcium signaling activity following dye injection while the remainder of the oocyte was quiescent. Fertilization triggered a localized elevation of free calcium in the cortex overlying the animal pole between 15-30 sec post insemination with little involvement of the underlying central cytoplasm. Next, calcium release in the cortex began to spread peripherally around the perimeter of the oocyte and by $8 \mathrm{mpi}$, the entire oocyte cortex exhibited elevated $\left[\mathrm{Ca}^{2+}\right]_{\text {i }}$. In contrast, the central cytoplasm did not exhibit a significant increase in free calcium until 3-4 mpi. Calcium release in the central cytoplasm progressed across the oocyte slowly and by 8 mpi, had involved the entire oocyte. To accurately analyze the calcium changes in different compartments of the oocyte, calcium green fluorescence was quantitated separately in the cortex and central cytoplasm using the region measurement capabilities of Metamorph software. The cortical region was drawn as an arc to include approximately $20 \%$ of the oocyte cortex centered over the initial site of calcium release. The central cytoplasm region was drawn as a circle encompassing approximately $25 \%$ of the cross sectional area of the oocyte. Both regions had to be relocated during the time series as the zygote moved as a result of chorion elevation and during cortical contractions. However, this analysis allowed detection of small, localized changes that were poorly represented in measurements from the entire oocyte. Quantitation of the cortical and central cytoplasmic fluorescence demonstrated the difference between calcium signaling in these two compartments of the oocyte as seen in Fig. 4. Calcium green fluorescence in the cortex over the animal pole (solid line) increased rapidly after fertilization with a rapid initial transient observed within the first $15-30 \mathrm{sec}$. This was followed by a higher amplitude transient that reached a maximum at 1-2 mpi then declined. The central cytoplasm, in contrast, exhibited very little response during the first $2 \mathrm{mpi}$. $\left[\mathrm{Ca}^{2+}\right]_{\mathrm{i}}$ in the central cytoplasm began to increase between 3-4 mpi and reached a maximum at 6-
$8 \mathrm{mpi}$. Clearly, the calcium signaling events in these two compartments exhibit different time courses.

\section{Calcium signaling in relation to the micropyle}

Examination of the earliest response to fertilization was performed with a 20X objective lens focused at the micropyle where the fertilizing sperm was expected to penetrate the chorion and fuse with the oocyte plasma membrane. The micropyle in the unfertilized oocyte appeared as a cone shaped depression in the chorion causing a similar depression in the cortical cytoplasm (Fig. 5A white arrow). Insemination with sperm and water resulted in slight retraction of the oocyte from the chorion in the region of the micropyle during the first $30 \mathrm{sec}$ (Fig. 5B) which was accompanied by a small increase in cortical $\left[\mathrm{Ca}^{2+}\right]_{\mathrm{i}}$ evident as more concentrated fluorescence just to the left of the micropyle. This was followed by a more substantial increase in cortical $\left[\mathrm{Ca}^{2+}\right]_{i}$ around the micropyle (Fig. $5 \mathrm{C}$ ) and further elevation of the chorion due to hydrostatic forces. As the chorion elevated, the position of the fertilizing sperm first became evident since a fertilization cone formed with the sperm attached at the apex of a slender process (Fig. 5C, black arrow). Interestingly, the fertilizing sperm was typically found at some distance from the cone shaped depression in the oocyte cortex caused by the micropyle and similar results were obtained by examination of fixed oocytes labeled with DAPI or ethidium homodimer to identify the sperm head (not shown). It appears that the sperm did not fuse with the plasma membrane immediately underlying the micropyle, but rather to a cell process that originated somewhat lateral to it. By $1.5 \mathrm{mpi}$, the fertilization cone had become prominent and cortical $\left[\mathrm{Ca}^{2+}\right]_{\mathrm{i}}$ continued to increase (Fig. 5D). While calcium green fluorescence demonstrated that $\left[\mathrm{Ca}^{2+}\right]_{i}$ was elevated in the cortex underlying the fertilization cone at this stage, the fertilization cone itself exhibited little calcium green fluorescence. By 2 mpi, calcium green fluorescence became much more intense at the animal pole exceeding the intensity at the original site of sperm- 
oocyte fusion. This region was identified as the animal pole by the fact that contraction of the yolky cytoplasm produced a clear zone (Fig. 5E, ${ }^{*}$ ) characteristic of the animal pole. In summary, the fertilizing sperm triggers a localized calcium transient at the micropyle, which spreads laterally to the animal pole where it becomes amplified and eventually propagated through the oocyte cortex.

\section{Role of the sperm and hypotonic activation in calcium signal- ing}

In zebrafish, exposure of the oocyte to hypotonic conditions as a result of spawning into fresh water causes changes in calcium signaling and oocyte morphology that are similar to that in fertilized oocytes, and are sufficient to trigger unsuccessful attempts at mitosis. When $\left[\mathrm{Ca}^{2+}\right]_{\mathrm{i}}$ was quantitated with luminescence imaging, no difference could be detected in the dynamics of calcium signaling between fertilized and parthenogenetically activated oocytes (Lee, et al.,1999). However, when $\left[\mathrm{Ca}^{2+}\right]_{i}$ was monitored by confocal fluorescence microscopy and fluorescence in the cortex was quantitated separately from that of the central cytoplasm (Fig. 6A), it was clear that activation in the absence of sperm resulted in a very slow calcium release in the oocyte cortex. In contrast, the calcium release in the central cytoplasm occurred with similar kinetics in fertilized and parthenogenetically activated oocytes. In the absence of a fertilizing sperm, the calcium release in the oocyte cortex proceeded at a rate very similar to that in the central cytoplasm rather than the fast response characteristic of fertilization.

As an initial step in determining the role of the sperm, we tested the effect of sperm cytosol by injecting a cytoplasmic extract prepared from zebrafish sperm. This experiment should detect whether sperm carry a specific signal to the oocyte such as the highly active PLC zeta carried by mammalian sperm (Swann, et al.,2006). Oocytes pre-loaded with calcium green dextran were injected with a calcium-free sperm cytoplasmic extract delivered into the center of the yolk mass. As seen in Fig. 6B, this triggered a calcium transient in the oocyte cortex but did not induce a transient in the central cytoplasm. Image analysis revealed that this cortical transient began at the micropyle/animal pole even though the sperm cytoplasm was injected into the center of the
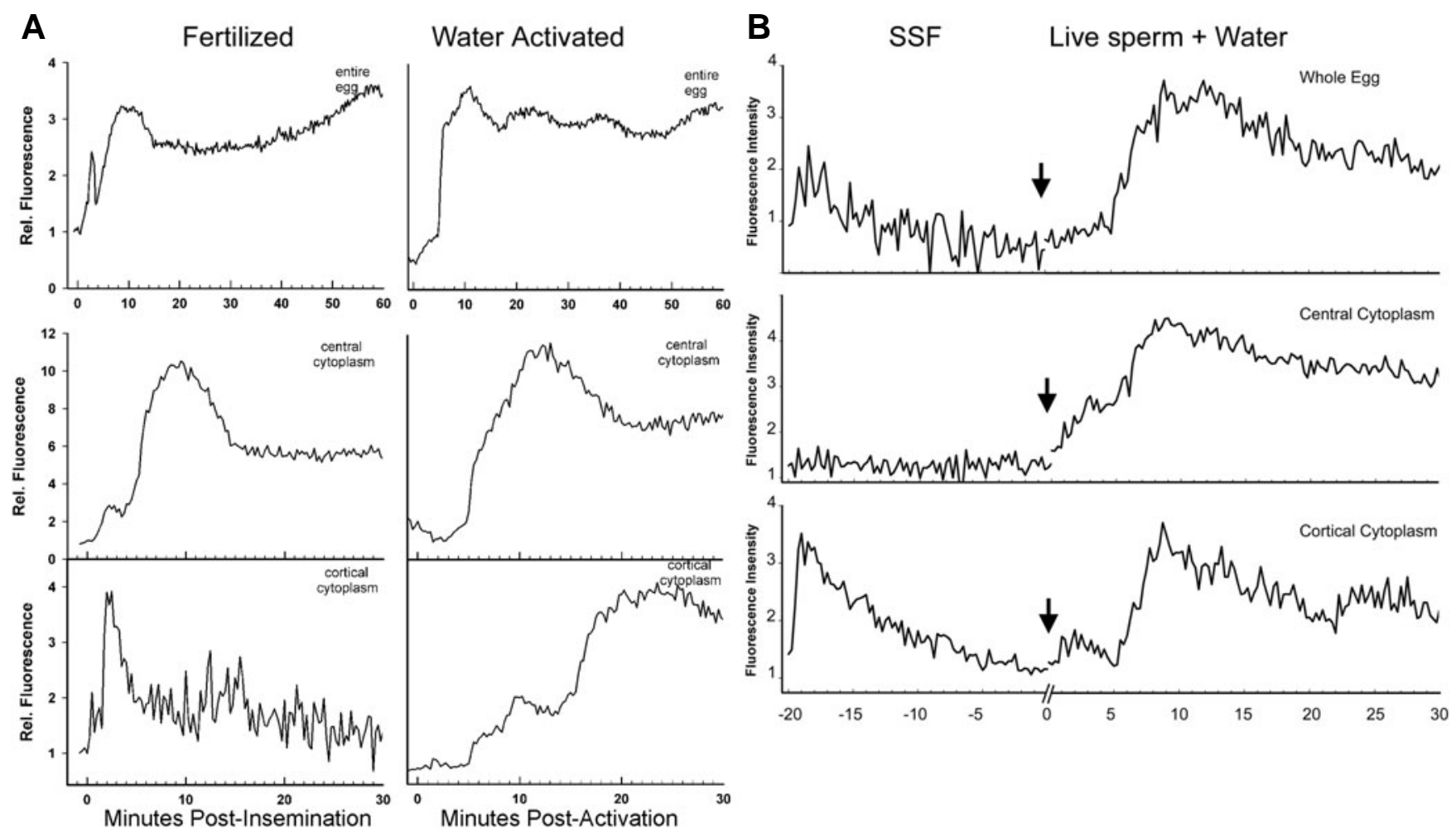

Fig. 6. Calcium signaling in response to hypotonic activation and soluble sperm factor. (A) Calcium release triggered by normal fertilization (left) was compared to that induced by hypotonic activation in the absence of sperm (right). Oocytes were injected with calcium green dextran and fluorescence was recorded at $15 \mathrm{sec}$ intervals following fertilization or hypotonic activation. The fluorescence intensity was measured across an image taken through the equator of the entire oocyte (top), in a region of central cytoplasm (middle) or in a region of cortical cytoplasm (lower panel). The oocyte fertilized by sperm exhibited a fast calcium transient in the oocyte cortex which reached a maximum within two to three minutes, while the oocyte activated by water exhibited a delayed cortical transient of lower amplitude. (B) The effect of soluble sperm cytoplasmic components was tested. An oocyte preloaded with calcium green dextran was injected with soluble sperm factor at time =-20 min. This resulted in a cortical calcium transient but no central cytoplasmic transient. The oocyte was then inseminated with sperm plus aquarium water (arrow) and recordings were resumed at time $=0$. Insemination triggered a second, cortical calcium transient of lower than normal amplitude that was followed by a normal appearing transient in the central cytoplasm. These results indicate that the fertilizing sperm triggers unique calcium responses in the egg cortex and that the calcium release in the central cytoplasm occurs without sperm. 
oocyte. Once the cortical transient had subsided, subsequent insemination with sperm and aquarium water triggered a normal appearing calcium release in the central cytoplasm, but the cortical calcium transient was weaker than normal, possibly reflecting desensitization of calcium release machinery following the first cortical transient.

Together, these results suggest that the contribution of the sperm to calcium signaling in the zebrafish oocyte may function to enhance the speed and amplitude of the calcium response in the oocyte cortex and we hypothesize that this might enhance the effectiveness of a block to polyspermy when sperm are present. Another implication of the above results is that the calcium signaling mechanism in the cortex is likely regulated in a manner different from that in the central cytoplasm.

\section{Biochemical characteristics of calcium signaling in the zebrafish oocyte}

Previous work in our laboratory demonstrated that fertilizationinduced activation of Src-family kinases such as Fyn played a significant role in regulating calcium release in the zebrafish
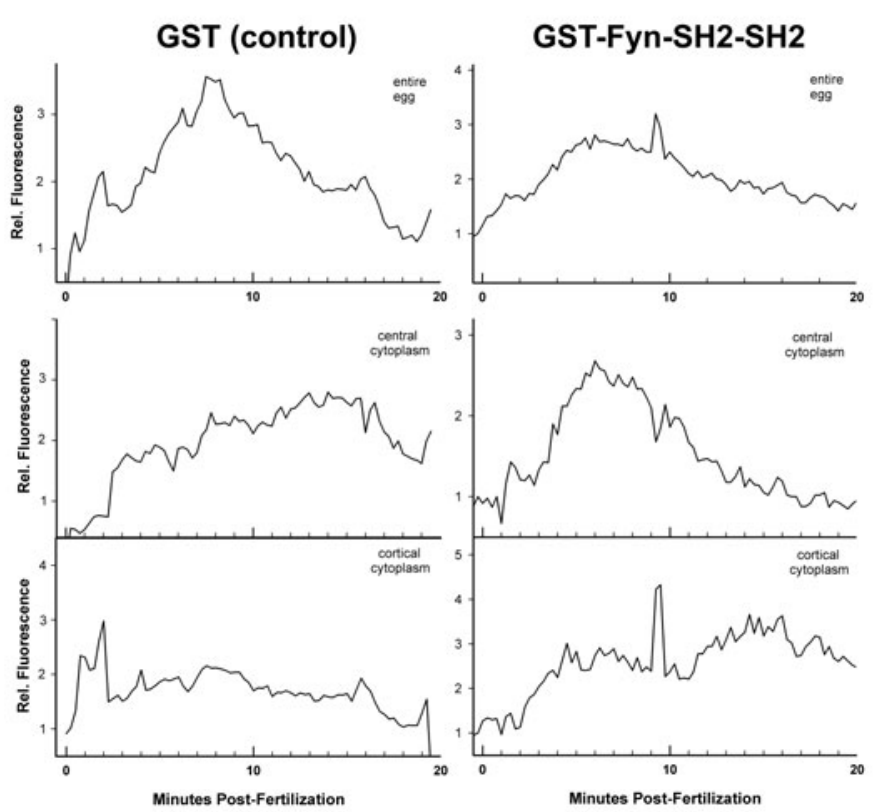

Fig. 7. Effect of a fusion protein containing Fyn $\mathrm{SH} 2$ domains on calcium green fluorescence at fertilization. Oocytes were injected with calcium green dextran and either GST (Control), or GST-Fyn-SH2$\mathrm{SH} 2$ at a final intracellular concentration of 5 to $6 \mu \mathrm{M}$, then images were recorded after a $10 \mathrm{~min}$ recovery period. Fertilization was accomplished by addition of a mixture of sperm and water at time $=0$ and images were recorded every $15 \mathrm{sec}$. The SH2-containing fusion protein suppressed the rapid cortical calcium release but had virtually no effect on calcium release in the central cytoplasm. Fluorescence was quantitated by pixel intensity quantitation using Metamorph 6.1 software. The top panels represent fluorescence measured from a cross section of the entire zygote. The middle panels represent fluorescence measured within a circular region in the center of the zygote (central cytoplasm) comprising approximately $25 \%$ of the total cross sectional area. The bottom panel represents fluorescence measured within an arc traced over the cortex (cortical cytoplasm) as near as possible to the micropyle. This arc comprised approximately $20 \%$ of the perimeter of the zygote. oocyte (Kinsey, et al., 2003). This result seemed to mimic previous findings in oocytes from Xenopus laevis, as well as marine invertebrate oocytes (Giusti, et al., 2000; Kinsey, and Shen, 2000b; Runft, and Jaffe, 2000). We have now used detailed confocal analysis to study the effect of Src-family PTK suppression on $\left[\mathrm{Ca}^{2+}\right]_{\mathrm{i}}$ signaling in the different compartments of the zebrafish oocyte. The approach was to use a SH2 domain fusion protein to suppress Src-family PTK signaling in the oocyte, then measure the dynamics of the fertilization-induced calcium transient in the oocyte cortex and central cytoplasm. In view of earlier work demonstrating the enhanced specificity of constructs containing tandem SH2 domains (Bradshaw, and Waksman, 2002), we constructed a GST fusion protein containing tandem $\mathrm{SH} 2$ domains from Fyn (GST-Fyn-SH2-SH2) and injected this prior to fertilization at a concentration similar to that used previously for GST-Fyn-SH2 (Wu and Kinsey, 2002). Oocytes injected with the control GST protein exhibited a normal cortical calcium transient reaching a maximum at approximately $2 \mathrm{mpi}$, and a slower transient in the central cytoplasm (Fig. 7). Oocytes injected with GST-Fyn-SH2-SH2 produced a delayed cortical calcium transient, with no high amplitude spike during the first two minutes (Fig. 7, bottom). However, the slow calcium transient in the central cytoplasm was relatively unaffected by GST-Fyn-SH2-SH2. This result supports the hypothesis that Fyn kinase signaling is required primarily for calcium events that occur in the cortical cytoplasm rather than the central cytoplasm.

\section{Localization of signaling components in the oocyte}

The observed differences in calcium signaling in regions of the zebrafish oocyte suggested that the signaling components responsible for the implementation and control of calcium release might be localized to specific regions of the oocyte. Confocal immunofluorescence localization of the IP3 receptor (IP3r), (Fig. 8) revealed that this channel protein was highly enriched in the cortical cytoplasm both before (Fig. 8A) and after fertilization (Fig. $8 B$ ). The distribution of IP3r in the oocyte cortex was initially fairly uniform with no evidence of enrichment at the animal pole. However, once blastodisc formation began (Fig. 8C) the IP3r became concentrated over the animal pole.

The distribution of Fyn kinase was also studied by confocal immunofluorescence. As seen in Fig. 9, Fyn kinase was highly enriched in the oocyte cortex, but the distribution within the cortex was not uniform and fluorescence intensity was consistently higher in the animal pole than over the vegetal pole (Fig. 9A). The region of the fertilization cone (Fig. 9B) was enriched in Fyn kinase as was typical of the animal pole region. Once blastodisc formation was underway, Fyn kinase became concentrated over the blastodisc as was observed for the IP3r. In summary, these results demonstrate that the localized features of calcium signaling in the zebrafish oocyte correlate well with the specialized distribution of IP3r and Fyn kinase since calcium release occurred faster in the oocyte cortex where the highest density of Fyn and IP3r occurred.

While the above experiment demonstrated that Fyn kinase was expressed in the cortex and was concentrated at the animal pole, the result did not address whether Fyn was activated in that subcellular compartment. We have recently used a phosphorylation site-specific antibody to detect activated Src-family PTKs by immunofluorescence and Western blot (Sharma and Kinsey, 
Fig. 8. Localization of IP3r in the zebrafish oocyte. Oocytes were fixed before (A) or at different times after fertilization; $2.5 \mathrm{mpi}$ (B,D), $45 \mathrm{mpi}(\mathbf{C})$. The fixed oocytes were then processed for immunofluorescence as described in 'Materials and Methods'. Oocytes were labeled with the anti-IP3r type 1 antibody $(\mathbf{A}, \mathbf{B}, \mathbf{C})$ at a concentration of $0.5 \mu \mathrm{g} / \mathrm{m} /$ or with normal rabbit IgG (D) as a control. Bound antibody was detected with goat anti-rabbit IgG conjugated with Alexa Fluor-488. Immunofluorescence analysis demonstrated that the IP3 receptor was initially distributed uniformly in the oocyte cortex with no concentration at the animal pole, but later became concentrated over the blastodisc evident in (C) as a bulge of clear cytoplasm.

Fig. 9. Localization of Fyn in the zebrafish oocyte. Oocytes were fixed before (A) or at different times after fertilization: $2.5 \mathrm{mpi}$ (B,D), $45 \mathrm{mpi}$ (C). The fixed oocytes were then processed for immunofluorescence. Oocytes were labeled with the anti-Fyn3 antibody $(\mathbf{A}, \mathbf{B}, \mathbf{C})$ at a concentration of $0.5 \mu \mathrm{g} / \mathrm{ml}$ or with Fyn3 antibody + the synthetic peptide antigen (1 $\mathrm{mM})$ as a control (D). Bound antibody was detected with goat anti-rabbit $\lg G$ conjugated with Alexa Fluor-488. Prior to and immediately after fertilization, the distribution of Fyn kinase differs from that of the IP3 receptor in that it is concen-
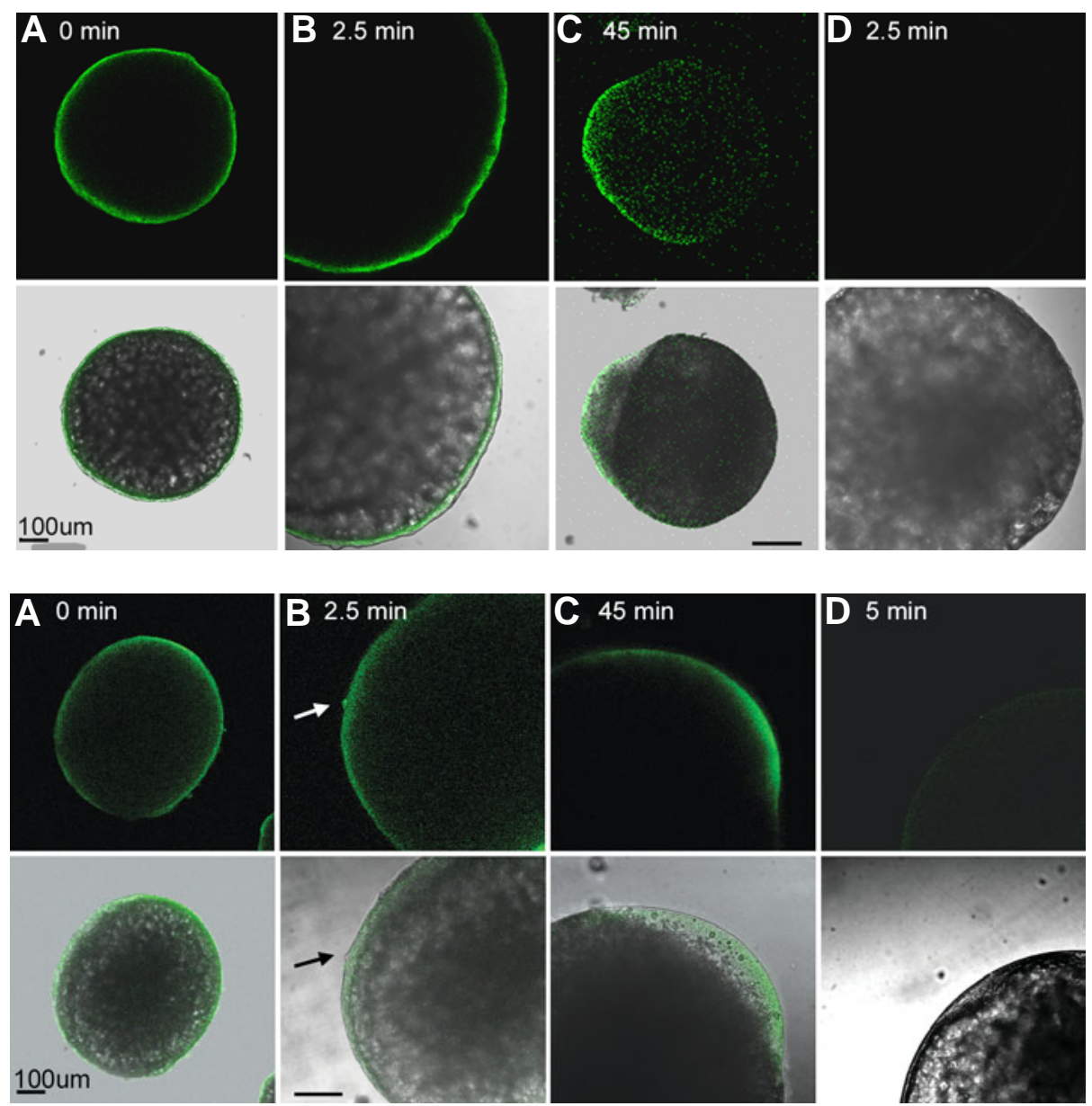
trated at the animal pole even before fertilization (A) and at the site of sperm fusion (white arrow, B). By 45 mpi (C), Fyn was localized over the blastodisc cortex and was usually concentrated within a smaller region of the blastodisc possibly indicating a region of intense signaling.

2006). Analysis of zebrafish oocytes fixed during the initial spermoocyte interactions (Fig. 10) revealed that Src-family PTKs (presumably including Fyn) are activated at the specific site of spermoocyte fusion as well as the adjacent components of the fertilization cone and surrounding cortical cytoplasm. This result is significant because it demonstrates that the initial interaction with the sperm caused kinase activation at the site of sperm-oocyte contact, but also that kinase activation was propagated laterally to deliver a signal to other regions of the oocyte cortex.

\section{Discussion}

The zebrafish oocyte resembles oocytes from other broadcast spawning vertebrates and invertebrates in that fertilization triggers a rapid, high amplitude calcium transient that is critical for oocyte activation. The polarized nature of the zebrafish oocyte defines the region of sperm-oocyte contact through the location of the micropyle that allows the sperm to penetrate the chorion. In addition to a basic structure that is polarized across the animal vegetal axis, the zebrafish oocyte includes a large compliment of yolk that comprises most of the central cytoplasmic mass of the oocyte leaving a thin layer of clear cytoplasm restricted to the oocyte cortex. Both of these compartments participate in the fertilization-induced calcium transient but previous studies dem- onstrated that calcium release propagated through the cortex much faster than through the central cytoplasm (Lee, et al.,1999). Our present studies have used confocal imaging to define more precisely the dynamics of calcium release within these two compartments and to correlate them with the distribution of signal transduction enzymes that regulate calcium release from internal stores.

By imaging the micropyle, we were able to pinpoint the site of sperm-oocyte contact and record the initial response to the fertilizing sperm. We observed that the sperm fuses with the oocyte at a short distance from the micropyle either by moving within the perivitelline space, or by fusing with a cell process that extends into the micropyle (Hart and Donovan, 1983). In any case, sperm binding or fusion is quickly $(15-30 \mathrm{sec})$ followed by a localized calcium release in the underlying cortical cytoplasm. This initial calcium release spreads to the adjacent animal pole where it becomes amplified in magnitude and then spread peripherally throughout the oocyte cortex reaching a peak amplitude at approximately $2 \mathrm{mpi}$. The calcium release throughout the cortex is initially more intense in the animal hemisphere, but eventually (8-10 mpi) becomes uniform at a lower level of intensity. The cortical calcium transient begins to spread into the central cytoplasm underlying the animal pole at $1-2 \mathrm{mpi}$ and slowly progresses across the oocyte reaching a maximum amplitude at 8-10 mpi. 
Fig. 10. Localization of activated Src-family PTKs in the zebrafish oocyte. Zebrafish oocytes were fixed at 1.5 mpi to capture the fertilization cone with attached sperm. They were then processed for immunofluorescence in the presence of phosphatase inhibitors and then incubated with the clone-28 antibody to label activated (dephosphorylated) Src-family PTKs $(0.5 \mathrm{ug} / \mathrm{ml})$. Bound antibody was detected with goat anti-mouse IgG conjugated to Alexa fluor-488. The fertilizing sperm is visible as a round spot at the apex of the fertilization cone which protrudes from the right hand edge of the oocyte. Src-family PTKs were strongly activated in the region of the fertilization cone indicating the response of the oocyte to fertilization. Magnification is indicated by the bar which represents $25 \mu \mathrm{m}$.
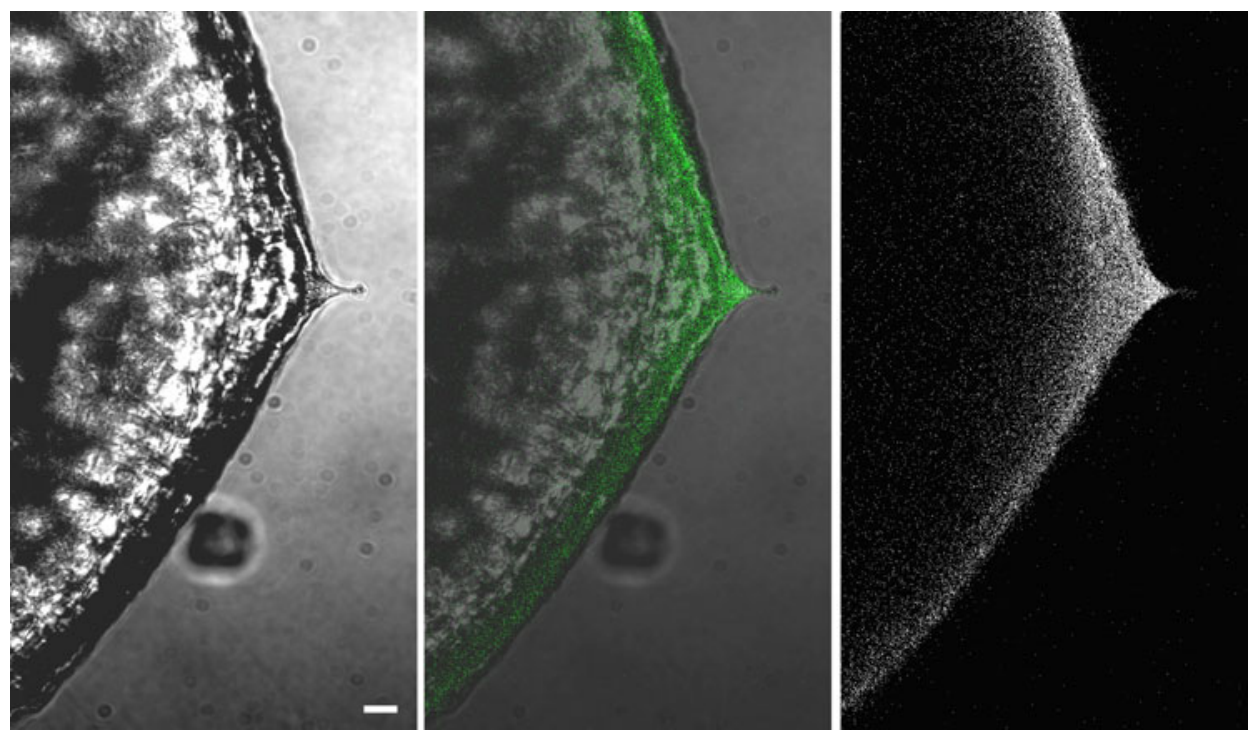

One possible explanation for this rather complicated process of calcium signaling may lie in the arrangement of the signaling components involved. Fyn kinase and the IP3r are highly enriched in the cortical cytoplasm and barely detectable in the central cytoplasm. Furthermore, Fyn kinase is enriched over the animal pole hemisphere and especially concentrated in the cortex of the presumptive animal pole while the IP3r appears to be uniformly distributed in the oocyte cortex. The strong amplification of the cortical calcium transient at the animal pole may be a consequence of intense Fyn kinase signaling at that site which would be expected to enhance PLC $\gamma$ activity and therefore the rate of IP3 production. Further study of PLC $\gamma$ distribution and phosphorylation may shed further light on this question.

Functional studies revealed further differences in calcium signaling within the oocyte cortex and central cytoplasm. The cortical calcium transient is not well established during parthenogenetic activation by hypotonic shock, although this treatment induces a normal appearing transient in the central cytoplasm. Microinjection of 'soluble sperm factor' containing sperm cytosol triggers a calcium transient in the oocyte cortex but not the central cytoplasm. Finally, injection of a fusion protein containing tandem $\mathrm{SH} 2$ domains of Fyn kinase suppresses the calcium transient in the oocyte cortex but has little effect on the transient in the central cytoplasm.

Our interpretation of the above results is that the zebrafish oocyte cortex is highly specialized to respond to the fertilizing sperm (possibly a soluble sperm factor) to produce a rapid, high intensity calcium transient in the cortex. This cortical transient requires Fyn kinase activity for optimal propagation and makes use of a concentration of IP3r in the cortex to allow maximal calcium release from the cortical endoplasmic reticulum. The concentration of Fyn kinase at the animal pole may facilitate amplification of the initial signal from the sperm. In fact, immunostaining with an antibody to activated Src-family PTKs demonstrates that Fyn (and possibly Src or Yes) kinase is activated locally at the site of sperm-oocyte contact and then progressively through the oocyte cortex. No such activation is detected in the central cytoplasm, which may be one of the reasons that calcium release proceeds so slowly through the central cytoplasm. Fish oocytes that are specialized to produce a rapid cortical calcium transient would have an advantage in preventing polyspermy due to a rapid cortical reaction, but the requirement for speed in the central cytoplasm may not exist. Calcium dynamics in the central cytoplasm of the zebrafish oocyte may instead be involved primarily with cell cycle control and can occur at a slower rate. The rapid, cortical calcium signaling in the zebrafish oocyte resembles the rapid calcium responses typical of oocytes from other broadcast spawning species such as sea urchins, starfish, and frogs in that it involves Src-family PTK activity. Mammalian oocytes exhibit much slower calcium transients and appear not to use Srcfamily PTKs as part of the calcium oscillation machinery. In any case, the fish oocyte represents an excellent model to study the initial sperm-oocyte interactions and future studies will hopefully characterize the signal delivered by the sperm, and the mechanism underlying the unique progressive activation of Fyn kinase in this model system.

\section{Materials and Methods}

\section{Buffers}

Injection buffer: $\mathrm{KCl}, 0.15 \mathrm{M} ; \mathrm{NaCl}, 3 \mathrm{mM} ; \mathrm{KH}_{2} \mathrm{PO}_{4}, 10 \mathrm{mM}(\mathrm{pH} 7.2)$; glutathione, $1 \mathrm{mM}$; sucrose, $80 \mathrm{mM}$; EGTA, $10 \mathrm{nM}$. Hank's BSA: NaCl, $137 \mathrm{mM}$; KCl, $5.4 \mathrm{mM} ; \mathrm{Na}_{2} \mathrm{HPO}_{4}, 0.25 \mathrm{mM} ; \mathrm{CaCl}_{2}, 1.37 \mathrm{mM}$; $\mathrm{MgSO}_{4}, 1.0$ $\mathrm{mM}$; $\mathrm{NaHCO}_{3}, 4.2 \mathrm{mM}$, pH 7.2; BSA (Sigma, St. Louis, MO.) $25 \mathrm{mg} / \mathrm{ml}$. Fixative: $\mathrm{Na}_{2} \mathrm{HPO}_{4}, 50 \mathrm{mM}$; sucrose, $4 \% \mathrm{wt} / \mathrm{vol}$; paraformaldehyde, $4 \%$ wt/vol; phenylarsine oxide, $40 \mu \mathrm{M}$; $\mathrm{pH}$ 7.2. Glycine blocking solution: $\mathrm{NaH}_{2} \mathrm{PO}_{4}, 50 \mathrm{mM}$; glycine, $10 \mathrm{mM}$; sucrose, $4 \%$ wt/vol; NP40, 0.5\% vol/ vol; goat serum blocking solution was identical to glycine blocking solution except that goat serum, $2 \% \mathrm{vol} / \mathrm{vol}$ replaced the glycine.

\section{Oocytes}

Oocytes were collected from mature Danio rerio and maintained in Hank's BSA buffer at $28^{\circ} \mathrm{C}$, while sperm were maintained on ice in sperm extender solution (Lee, K. W. et al.,1999). Fertilization was accomplished by mixing the sperm $(5 \mu \mathrm{l})$ with the oocytes, then activating the sperm by addition of $2.5 \mathrm{ml}$ of aquarium water.

\section{Microinjection and calcium imaging}

Oocytes were immobilized either with an oocyte injection chamber (Lee, K. W. et al.,1999) or with a suction pipette and maintained in a 
quiescent state in Hank's-BSA. Oocytes were injected using glass micropipettes beveled at $30^{\circ}$ with a tip diameter of $2.5 \mu \mathrm{m}$. The pipettes were back loaded with $1-2 \mu \mathrm{l}$ of test solution and $10 \mu \mathrm{l}$ of mineral oil. Injection volumes (usually 1-2 $\mathrm{nl}$ ) were calculated after oocyte injection by injecting representative amounts of each test solution into mineral oil, then measuring the diameter of the droplet. In order to detect developmental changes in $\left[\mathrm{Ca}^{2+}\right]_{\mathrm{i}}$, oocytes were injected with calcium green-dextran to achieve intracellular concentrations of approximately $0.5 \mu \mathrm{M}$. The oocytes were then monitored by confocal fluorescence microscopy on a Nikon TE2000U microscope using a 488nm Spectra Physics (Mountainview, CA) laser. Time course measurements were made with a $4 \mathrm{X}$ or $20 \mathrm{X}$ super fluor objective with pinhole settings set to obtain a theoretical $30 \mu \mathrm{m}$ optical section through the equator of the embryo. Emitted fluorescence was recorded at $15 \mathrm{sec}$ intervals and separate images were collected using transmitted light to obtain a DIC image and a $515 \mathrm{~nm} / 30 \mathrm{~nm}$ band pass filter to obtain calcium green fluorescence. The fluorescence intensity of the zygote was quantitated from digital images by centering an elliptical measurement area over the entire zygote, a second cortical region approximately $30 \mu \mathrm{m}$ thick was traced to include approximately $20 \%$ of the oocyte cortex nearest the micropyle, and a third region including approximately $25 \%$ of the area of the central cytoplasm. A fourth area including adjacent medium was also recorded to control for changes in background fluorescence. Average pixel density was calculated by Metamorph 6.1, (Universal Imaging Corp., Downington, PA).

\section{Preparation of soluble sperm factor}

Sperm were collected from twenty male fish and maintained in sperm extender solution. The protein content was determined and the sperm were concentrated by centrifugation at 10,000XG for 5minutes. They were then resuspended in $100 \mu \mathrm{l}$ of injection buffer and washed by centrifugation twice then suspended at a final concentration of $10 \mathrm{mg}$ protein $/ \mathrm{ml}$ of injection buffer. The sperm were lysed by brief sonication with a probe sonicator and the particulate fraction was removed by centrifugation in an ultracentrifuge at $100,000 \times \mathrm{G}$ for 30 minutes. The resulting 'sperm soluble factor' contained the cytosol from $200 \times 10^{6}$ sperm per ml.

\section{Preparation of GST fusion proteins}

A GST fusion protein encoding tandem copies of the $\mathrm{SH} 2$ domain of Fyn kinase was constructed by PCR amplification of the Xenopus Fyn $\mathrm{SH} 2$ domain using primers encoding an EcoR 1 site at the $\mathrm{N}$-terminus and an Xho-1 site at the C-terminus (Kinsey, and Shen, 2000a). The amplified sequence was self-ligated using a linker containing an EcoR1 site and Xho1 site (produced by hybridization of 5' TCGAGTCGACCCGGG3' and 5'AATTCCCGGGTCGAC3') to generate a tandem SH2 domain. This was ligated to GST and expressed as a fusion protein, and purified as previously described (Kinsey and Shen, 2000b). Fusion proteins were dialyzed into injection buffer and adjusted to $15 \mathrm{mg} / \mathrm{ml}$ protein.

\section{Immunofluorescence}

Zebrafish oocytes and zygotes were fixed for immunofluorescence in a formaldehyde fixative containing the irreversible phosphatase inhibitor phenylarsine oxide to prevent dephosphorylation of SFKs during the immuno-staining process. Phenylarsine oxide was present during all manipulations of oocytes up to the actual microscopy when it was replaced with sodium orthovanadate which was less likely to interact with UV light. Fixation was carried out overnight at $4^{\circ} \mathrm{C}$, then the oocytes were washed into phosphate buffered saline (PBS) and pronase $0.5 \% \mathrm{wt} / \mathrm{vol}$ (Calbiochem, San Diego, CA) for $6 \mathrm{~min}$ at $25^{\circ} \mathrm{C}$ to permeabilize the chorion. Zygotes fixed more than $60 \mathrm{sec}$ post-insemination also required manual dissection of the then hardened chorion. Fixed oocytes were permeabilized with glycine blocking solution containing $0.5 \%$ NP40 for 1 $\mathrm{h}$ at $25^{\circ} \mathrm{C}$, then washed into goat serum blocking buffer for at least $2 \mathrm{~h}$. Antibody dilutions were made in goat serum blocking buffer and oocytes were incubated overnight at $4^{\circ} \mathrm{C}$. The primary antibody used included affinity purified rabbit anti-Fyn3 (Santa Cruz Biotechnology, Santa Cruz, CA), rabbit anti IP3r type 1 (Sigma Chemical Co., St. Luis, MO), or mouse Clone 28 (Biosource International. Camarillo, CA). Secondary antibodies were applied after four washings with goat serum blocking buffer and oocytes were incubated with the secondary antibody for 90 minutes at $25^{\circ} \mathrm{C}$. The secondary antibody was goat anti-rabbit IgG-Alexa-fluor 488 or goat-anti-mouse IgG-Alexa-fluor 488 (In Vitrogen, Carlsbad, CA). The oocytes were then monitored by confocal fluorescence microscopy on a Nikon TE2000U microscope using a 488nm Spectra Physics (Mountainview, CA) laser.

\section{Acknowledgments}

This work was supported by a grant from NICHD HD-14846 to W.H.K. We would like to acknowledge the technical assistance of Cory Lewis during this study. We also would like to acknowledge the intellectual contributions of D. Garbers who was the first to demonstrate PTK activation in the response of the oocyte to fertilization and whose focus on biochemical analysis of fertilization was an important inspiration for this study.

\section{References}

BRADSHAW, J. M. and WAKSMAN, G.(2002). Molecular recognition by $\mathrm{SH} 2$ domains. Advances in Protein Chemistry 61:161-210.

CARROLL, D. J., RAMARAO, C. S., MEHLMANN, L. M., ROCHE, S., TERASAKI, M., and JAFFE, L. A.(1997). Calcium release at fertilization in starfish eggs is mediated by phospholipase Cgamma. J-Cell-Biol. 138:1303-1311.

CARROLL, J.(2001).The initiation and regulation of $\mathrm{Ca} 2+$ signalling at fertilization in mammals. Semin. Cel/ Dev Bio/12:37-43.

CHANG, D. C. and MENG, L.(1995). A localized elevation of cytosolic free calcium is associated with cytokinesis in the zebrafish embryo. J-Cell-Biol. 131:15391545.

CRETON, R., SPEKSNIJDER, JE, and JAFFE, LE. (1998). Patterns of free calcium in zebrafish embryos. J.Cel/ Science 111:1613-1622.

GALIONE, A., MCDOUGALL, A., BUSA, W. B., WILLMOTT, N., GILLOT, I., and WHITAKER, M. (1993).Redundant mechanisms of calcium-induced calcium release underlying calcium waves during fertilization of sea urchin eggs. Science 261:348-352.

GILKEY, J. C., JAFFE, L. F., RIDGWAY, E. B., and REYNOLDS, G. T. (1978).A free calcium wave traverses the activating egg of the medaka, Oryzias latipes. Journal of Cell Biology 76:448-466.

GIUSTI, A. F., XU, W. Q., HINKLE, B., TERASAKI, M., and JAFFE, L. A. (2000). Evidence that fertilization activates starfish eggs by sequential activation of a Src-like kinase and phospholipase Cgamma. Journal of Biological Chemistry 275:16788-16794.

HART, N. H. and DONOVAN, M. (1983).Fine Structure of the chorion and site of sperm entry in the egg of brachydanio. Journal of Experimental Zoology 227:277-296.

IWAMATSU, T., YOSHIMOTO, Y., and HIRAMOTO, Y.(1988a). Cytoplasmic Ca2+ release induced by microinjection of $\mathrm{Ca} 2+$ and effects of microinjected divalent cations on $\mathrm{Ca} 2+$ sequestration and exocytosis of cortical alveoli in the medaka egg. Dev Bio/125:451-457.

IWAMATSU, T., YOSHIMOTO, Y., and HIRAMOTO, Y.(1988b). Mechanism of $\mathrm{Ca} 2+$ release in medaka eggs microinjected with inositol 1,4,5-trisphosphate and Ca2+. Dev Biol129:191-197.

KINSEY, W. H. and SHEN, S. S.(2000a). Role of the Fyn kinase in calcium release during fertilization of the sea urchin egg. Dev Biol. 225:253-264.

KINSEY, W. H. and SHEN, S. S.(2000b). Role of the Fyn kinase in calcium release during fertilization of the sea urchin egg. Devel.Biol. 225:253-264.

KINSEY, W. H., WU, W., and MACGREGOR, E.(2003). Activation of Src-family PTK activity at fertilization: role of the SH2 domain. Dev Biology 264:255-262.

LEE, K. W., WEBB, S. E., and MILLER, A. L.(1999). A wave of free cytosolic calcium traverses zebrafish eggs on activation. Dev Biol. 214:168-180.

LEE, K. W., WEBB, S. E., and MILLER, A. L.(2003). Ca2+ released via IP3 receptors is required for furrow deepening during cytokinesis in zebrafish 
embryos. Dev Biol. 47:411-421.

RUNFT, L. L. and JAFFE, L. A.(2000). Sperm extract injection into ascidian eggs signals $\mathrm{Ca}^{2+}$ release by the same pathway as fertilization. Development127:32273236.

RUNFT, L. L., JAFFE, L. A., and MEHLMANN, L. M. (2002).Egg activation at fertilization: Where it all begins. Dev Biol. 245:237-254.

SHARMA, D. and KINSEY, W. H.(2006). Fertilization triggers localized activation of Src-family protein kinases in the zebrafish egg. Dev Biol. 295:604-614.

SWANN, K., LARMAN, M. G., SAUNDERS, C. M., and LAI, F. A.(2004).The cytosolic sperm factor that triggers $\mathrm{Ca} 2+$ oscillations and egg activation in mammals is a novel phospholipase C: PLCzeta. Reproduction. 127:431-439.

SWANN, K., SAUNDERS, C. M., ROGERS, N. T., and LAI, F. A.(2006). PLCzeta(zeta): a sperm protein that triggers $\mathrm{Ca} 2+$ oscillations and egg activa- tion in mammals. Semin. Cell Dev.Biol. 17:264-273.

WEBB, S. E., LEE, K. W., KARPLUS, E., and MILLER, A. L.(1997). Localized calcium transients accompany furrow positioning, propagation, and deepening during early cleavage period of zebrafish embryos. Dev Biol. 192:78-92.

WU, W. and KINSEY, W. H.(2002). Role of PTPase(s) in regulating Fyn kinase at fertilization of the zebrafish egg. Dev Biol. 247:286-294.

YAMAMOTO, T.(1954). Physiological studies on fertilization and activation of fish eggs. V. The role of calcium ions in activation of Oryzias eggs. Exp Cell Res 6:56-68.

YOSHIMOTO, Y. and HIRAMOTO, Y.(1988). Temporal and spatial changes in intracellular $\mathrm{Ca} 2+$ upon fertilization and during cell division. Tanpakushitsu Kakusan Koso 33:2292-2299.

\section{Related, previously published Int. J. Dev. Biol. articles}

See our recent Special Issue Ear Development edited by Fernando Giraldez and Bernd Fritzsch at: http://www.ijdb.ehu.es/web/contents.php?vol=51\&issue=6-7

Defective calcium release during in vitro fertilization of maturing oocytes of LT/Sv mice Karolina Archacka, Anna Ajduk, Pawel Pomorski, Katarzyna Szczepanska, Marek Maleszewski and Maria A. Ciemerych Int. J. Dev. Biol. (2008) 52: doi: 10.1387/ijdb.072397ka

\section{Expression of protocadherin 18 in the CNS and pharyngeal arches of zebrafish embryos} Fumitaka Kubota, Tohru Murakami, Yuki Tajika and Hiroshi Yorifuji Int. J. Dev. Biol. (2008) 52: 397-405

Cadherin- 6 is required for zebrafish nephrogenesis during early development Fumitaka Kubota, Tohru Murakami, Kenji Mogi and Hiroshi Yorifuji Int. J. Dev. Biol. (2007) 51: 123-129

Skin development in bony fish with particular emphasis on collagen deposition in the dermis of the zebrafish (Danio rerio). Dominique Le Guellec, Ghislaine Morvan-Dubois and Jean-Yves Sire Int. J. Dev. Biol. (2004) 48: 217-231

Ca2+-independent protein kinase $\mathbf{C}$ signalling in mouse eggs during the early phases of fertilization. Carla Tatone, Simona Delle Monache, Antonella Francione, Luisa Gioia, Barbara Barboni and Rosella Colonna Int. J. Dev. Biol. (2003) 47: 327-333

Fertilization triggers activation of Fyn kinase in the zebrafish egg. W Wu and W H Kinsey Int. J. Dev. Biol. (2000) 44: 837-841

Intestinal fatty acid binding protein gene expression reveals the cephalocaudal patterning during zebrafish gut morphogenesis.

M André, S Ando, C Ballagny, M Durliat, G Poupard, C Briançon and P J Babin Int. J. Dev. Biol. (2000) 44: 249-252

Parthenogenetic activation of mouse oocytes using calcium ionophores and protein kinase $\mathbf{C}$ stimulators.

$\mathrm{J}$ A Uranga, R A Pedersen and J Arechaga

Int. J. Dev. Biol. (1996) 40: 515-519

Mechanisms of initiation and propagation of the calcium wave during fertilization in deuterostomes.

F Berger

Int. J. Dev. Biol. (1993) 37: 245-262

Calcium in sea urchin egg during fertilization.

I Gillot, P Payan, J P Girard and C Sardet

Int. J. Dev. Biol. (1990) 34: 117-125

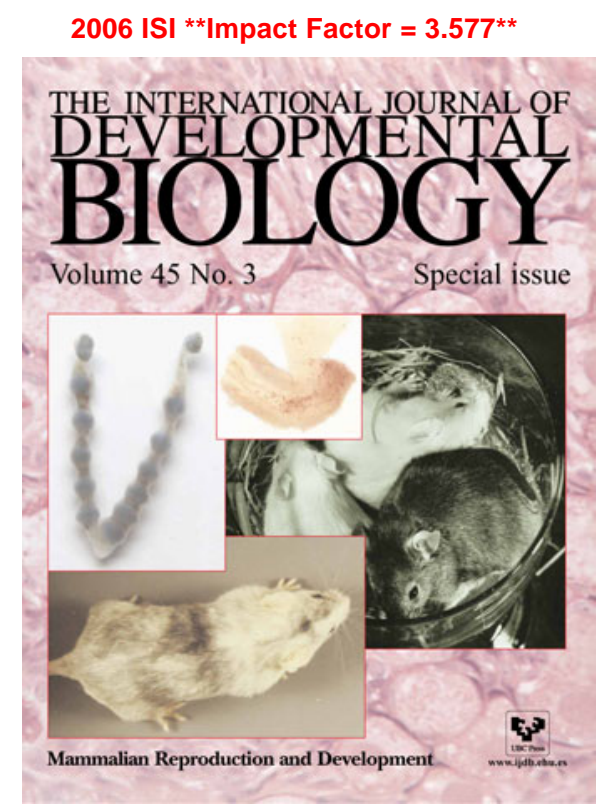

